\title{
Inhalt
}

\section{Editorial -6}

Michael Braum

Worauf baut die Bildung? - Anforderungen an eine Bildungsbaukultur _ 8

Michael Braum, Bernhard Heitele. Kommentiert von Oliver G. Hamm

Wir waren schon mal weiter - Betrachtungen zu sich wandelnden Prämissen im Schulbau 16 Arno Lederer

Zurück in die Zukunft - Auszüge aus einem Podiumsgespräch im Mai $2009 \_22$

Positionen

Wie werden unsere Kinder lernen? - Acht Thesen zur Schule der Zukunft 26 Otto Seydel

Schulraum ist Lebensraum - Der Schüler hat das Maß aller Dinge zu sein _ 36 Falk Jaeger

Warum sind die anderen besser? Schul(bau)entwicklung in der Schweiz, in den Niederlanden und in Finnland 42

Barbara Pampe

Vorbildlich - Beispiele für Bildungsbauten 52

Oliver G. Hamm / Fotografien: Petra Steiner

Betriebskita der Metro Group, Düsseldorf __54 / Kindertagesstätte Griechische Allee, Berlin _ 58 /

Kindertagesstätte „Plappersnut“, Wismar_ 62 / Katharinenschule, Hamburg _ 66 / Grundschule Helsinkistraße, München _ 70 /

Martinszentrum Bernburg _ 74 / Grundschule Schulzendorf _ 78 / Erika-Mann-Grundschule, Berlin _ 82 /

Internationale Friedensschule Köln _ 86 / Evangelisches Gymnasium Bad Marienberg _ 90 /

Schülerhaus des Friedrich-Eugens-Gymnasiums, Stuttgart__94 / Schulzentrum Schreienesch, Friedrichshafen __ 98 /

Hilde-Domin-Schule, Herrenberg _ 102 / Aula der Max-Taut-Schule, Berlin _ 106 / Domsingschule Stuttgart _ 110

Chancen und Grenzen - Prozess- und Gestaltqualität von Bildungsbauten _ 114

Oliver G. Hamm und Carl Zillich im Gespräch mit Cornelia von Ilsemann und Gerhard Kramer

Lebenslänglich verortet - Vom Wert des Lernens 122

Gert Kähler

Herausforderung Bildung 130

Michael Braum

Autoren -134

Bildnachweis 